\title{
DETERMINAÇÃO DE ARSÊNIO EM ÁGUAS CONTAMINADAS USANDO FLUORESCÊNCIA DE RAIOS-X POR ENERGIA DISPERSIVA
}

Luiz Carlos M. Pataca*, Gisele G. Bortoleto e Maria Izabel M. S. Bueno

Instituto de Química, Universidade Estadual de Campinas, CP 6154, 13084-971 Campinas - SP

Recebido em 8/3/04; aceito em 19/11/04; publicado na web em 13/4/05

\begin{abstract}
DETERMINATION OF ARSENIC IN CONTAMINATED WATERS USING ENERGY DISPERSIVE X-RAY FLUORESCENCE. This work proposes a simple, fast and inexpensive method to determine As in natural waters, using X-ray fluorescence. $50 \mu \mathrm{L}$ of each sample containing $100 \mathrm{mg} \mathrm{L}^{-1}$ of yttrium as internal standard were deposited over a $2.5 \mu \mathrm{m}$ thickness Mylar ${ }^{\mathrm{TM}}$ film. The samples were dried at $50{ }^{\circ} \mathrm{C}$ for $2 \mathrm{~h}$. X-ray spectra were obtained using an EDXRF apparatus. The accuracy was determined by analyte addition/recovery and by comparison with Hydride Generation Atomic Absorption Spectrometry (HG AAS). A recovery of about $100 \%$ was obtained and the results were in good agreement with HG AAS. The method showed a relative standard deviation of $6.8 \%$ and a detection limit of $10.5 \mu \mathrm{g} \mathrm{L}-1$ of As.
\end{abstract}

Keywords: arsenic; natural water; EDXRF.

\section{INTRODUÇÃO}

O arsênio, isolado em 1250 por Albertus Magnus, tem sido motivo de controvérsias durante toda a história da humanidade ${ }^{1}$. Na forma metálica e com elevado grau de pureza, é empregado na indústria eletrônica para produção de diodos e compostos semicondutores. Alguns compostos do elemento são empregados na agricultura, como matéria prima para produção de pesticidas. Na indústria química, é empregado como agente descolorante e espessante na produção de vidros e na purificação eletrolítica do zinco. $\mathrm{Na}$ medicina, é usado em algumas formulações de uso médico e veterinário e compostos de As, como o clorodietilarsênio e a Lewisita $\left(\mathrm{Cl}-\mathrm{CH}=\mathrm{CH}-\mathrm{AsCl}_{2}\right)$, que já foram utilizados como armas químicas no passado ${ }^{2,3}$.

A toxidez do elemento depende muito de sua forma química e de seu estado de oxidação ${ }^{1}$. O arsênio elementar não é tóxico, mas é rapidamente convertido a produtos tóxicos pelo organismo humano. A maior parte dos compostos contendo arsênio, sejam eles orgânicos ou inorgânicos, penta- ou trivalentes, acabam sendo convertidos pelo organismo ao trióxido de arsênio, o qual reage muito rapidamente com os grupos sulfidrilas (-SH) de proteínas, inibindo a ação enzimática e bloqueando a respiração celular ${ }^{4}$.

A introdução do arsênio no meio ambiente, especialmente em sistemas aquáticos, tem várias fontes, as quais podem ser de origem natural e antropogênica. As fontes naturais de contaminação por arsênio abrangem minerais e rochas que contêm As e os solos e sedimentos formados a partir dessas rochas, assim como os fenômenos geotermais e vulcânicos. As fontes antropogênicas incluem atividades relacionadas à preservação de madeira, à utilização do elemento na agricultura (geralmente em pesticidas), aos rejeitos provenientes da mineração, das atividades de refino dos metais não ferrosos e da queima de carvão, rico em $\mathrm{As}^{5}$.

No Brasil, as fontes naturais de contaminação por As, identificadas até o momento, estão relacionadas às rochas que hospedam depósitos auríferos sulfetados, como as da região do Quadrilátero

*e-mail: luizpataca@iqm.unicamp.br
Ferrífero (MG), as da Fazenda Brasileiro (Teofolândia-BA), as da Mina III (Crixás, GO) e as do Vale do Ribeira (SP). As fontes antropogênicas já identificadas no Brasil são pontuais e estão relacionadas com atividades de mineração e refino de minério de alguns dos depósitos auríferos acima mencionados. Só o Quadrilátero Ferrífero respondeu pela produção de $1.300 \mathrm{t}$ de $\mathrm{Au}$ nos últimos três séculos e, considerando a razão As/Au nos minérios, estima-se que pelo menos $390.000 \mathrm{t}$ de As devem ter sido liberadas para o ambiente ${ }^{6}$. Dessa forma, fica evidente que a região pode apresentar problemas de contaminação com o elemento.

Em Bangladesh, por ex., a contaminação de água por arsênio tem ameaçado a saúde pública de milhões de pessoas. Inúmeros casos de lesões na pele já foram diagnosticados, enquanto casos de câncer de pele e alguns outros tipos de câncer ainda estão sendo investigados e correlacionados com o alto teor de arsênio nas águas da região ${ }^{7}$.

Assim, é de grande importância o desenvolvimento de métodos analíticos eficientes, apropriados para análises de rotina e adequados para monitoração do elemento em regiões com prováveis contaminações pelo mesmo.

Segundo a Agência Nacional de Vigilância Sanitária (ANVISA) ${ }^{8}$, as metodologias analíticas para determinação dos parâmetros químicos devem atender às especificações das normas nacionais que disciplinam a matéria, da edição mais recente da publicação Standard Methods for the Examination of Water and Wastewater, de autoria das instituições "American Public Health Association" (APHA), ou das normas publicadas pela ISO ("International Standartization Organization"). De acordo com o método da $\mathrm{APHA}^{9}$, a espectrometria de absorção atômica com geração de hidretos (HG AAS) é recomendada para determinação de As, a qual tem se mostrado eficiente para quantificação deste elemento em águas, embora requeira procedimentos relativamente laboriosos.

A técnica de fluorescência de raios $\mathrm{X}$ por energia dispersiva (EDXRF), embora não atinja limites de detecção comparáveis aos alcançados pelas técnicas de espectrometria de absorção atômica, possui grandes vantagens como o baixo custo de análise, geralmente requer baixo consumo de reagentes e vidraria, gera pouco ou nenhum resíduo, o que também a torna ideal para se trabalhar 
em análises de rotina. Além disso, também pode ser considerada adequada para determinação de arsênio em amostras contaminadas, uma vez que essas amostras trazem o analito em concentrações geralmente acima da permitida pela $\mathrm{ANVISA}^{8}$, que atualmente fixa como valor máximo $10 \mu \mathrm{g} \mathrm{L}^{-1}$ de As em águas potáveis. Nesse caso, a técnica de EDXRF pode ser explorada para essa finalidade, mostrando-se como mais uma alternativa para quantificação de elementos em amostras líquidas ${ }^{10-12}$. Assim, no presente trabalho, foi desenvolvido um método utilizando a técnica em questão, para determinação de As em águas, após simples secagem da amostra sobre uma membrana apropriada.

O método foi aplicado para análise de amostras de águas provenientes da região do Quadrilátero Ferrífero - MG, as quais se apresentaram, muitas vezes, altamente contaminadas pelo analito.

\section{PARTE EXPERIMENTAL}

Todos os reagentes utilizados foram de grau analítico. A solução estoque de As (III) $1000 \mathrm{mg} \mathrm{L}^{-1}$ foi preparada pela diluição de uma ampola Tritisol ${ }^{\circledR}$ (Merck) em água desionizada e o volume da solução foi completado com água em um balão volumétrico de $1000 \mathrm{~mL}$. As soluções de trabalho foram preparadas diariamente, por diluição da solução estoque de arsênio, utilizando-se micropipeta Gilson ${ }^{\circledR}$.

O procedimento otimizado para o preparo da amostra baseiase na secagem de $50 \mu \mathrm{L}$ da solução da amostra, contendo $1 \mathrm{mg} \mathrm{L}^{-1}$ de ítrio como padrão interno, sobre um filme de Mylar $^{\circledR}$ de 2,5 $\mu \mathrm{m}$ de espessura, esticado no fundo de uma cela de polietileno com $32 \mathrm{~mm}$ de diâmetro externo e $23 \mathrm{~mm}$ de altura, com capacidade para $3 \mathrm{~mL}$ de amostra, marca Chemplex Industrties Inc., referência 1330.

As amostras foram secadas em estufa a $50{ }^{\circ} \mathrm{C}$ por $2 \mathrm{~h}$ e em seguida irradiadas, utilizando-se a técnica EDXRF. As irradiações foram realizadas em um Espectrômetro de Fluorescência de Raios $\mathrm{X}$, marca Shimadzu, modelo EDX-700, equipado com tubo de raios $\mathrm{X}$ de Rh. As seguintes condições de operação do equipamento foram selecionadas: tensão do tubo $-50 \mathrm{kV}$, corrente no tubo $500 \mu \mathrm{A}$, colimador $-10 \mathrm{~mm}$, tempo real de integração $-300 \mathrm{~s}$, tempo morto do detector $-1 \%$, sob vácuo. Como parâmetro de medida, utilizou-se a razão da área do pico do As (linha $K \alpha$ ) e da área do pico do Y (linha $\mathrm{K} \alpha$ ), através da análise relativa com curvas analíticas. As áreas dos picos foram determinadas utilizando o programa AXIL versão $3.5^{13}$.

As amostras de água analisadas nesse trabalho foram provenientes da região do Quadrilátero Ferrífero, situado em Minas Gerais, que têm apresentado problemas de contaminação por arsênio. A coleta de algumas das amostras foi feita seguindo as recomendações da CETESB ${ }^{14}$, enquanto outras foram doadas pelo Instituto de Geociências (IG-UNICAMP). Os procedimentos para as análises das amostras de água por HG AAS estão descritos na literatura ${ }^{15,16}$.

\section{RESULTADOS E DISCUSSÃO}

\section{Secagem da amostra}

Considerando que o método proposto baseia-se na pré-concentração do As por evaporação de solvente, testaram-se três modos de secagem da gota de $50 \mu \mathrm{L}$ pipetada sobre o filme esticado na cela: secagem à temperatura ambiente e ao ar, secagem à temperatura ambiente em dessecador e secagem à temperatura de $50{ }^{\circ} \mathrm{C}$ em estufa. Observou-se que não ocorria diferença entre os três modos de secagem testados. Assim, foi escolhido o método com aquecimento a $50{ }^{\circ} \mathrm{C}$ em estufa, por $2 \mathrm{~h}$, por ser o mais rápido.

\section{Interferência causada por íons cloreto}

Geralmente, amostras coletadas para determinação de arsênio utilizando a técnica de Espectrometria de Absorção Atômica com Geração de Hidretos (HG AAS) são preservadas em ácido clorídrico. Ácidos mais oxidantes, como $\mathrm{HNO}_{3}$, por ex., podem oxidar parte do As(III) a As(V), cujas intensidades dos sinais são diferentes. Assim, inicialmente, as amostras foram preservadas em $2 \%$ $\mathrm{HCl}(\mathrm{m} / \mathrm{v})$, ou seja, em $0,55 \mathrm{~mol} \mathrm{~L}^{-1}$ de $\mathrm{HCl}$. Durante os primeiros testes, verificou-se que em alguma etapa do método estava ocorrendo perda de arsênio e, muitas vezes, nenhum sinal do analito foi observado. Considerando-se que o composto $\mathrm{AsCl}_{3}$ que tem ponto de ebulição de $63{ }^{\circ} \mathrm{C}^{17}$, pudesse estar sendo formado, concluiu-se que o analito poderia estar sendo perdido sob essa forma. Nesse caso, essas perdas estariam ocorrendo durante a etapa de secagem da amostra.

Deste modo, as amostras passaram a ser preparadas em meio de $\mathrm{HNO}_{3} 2 \%(\mathrm{~m} / \mathrm{v})$, ou seja, 0,32 mol L-1. Após a alteração do meio, testou-se a interferência dos íons cloreto, na presença de até $2 \%(\mathrm{~m} / \mathrm{v})$ de cloreto, que corresponde à concentração de ácido clorídrico de $0,55 \mathrm{~mol} \mathrm{~L}^{-1}$, utilizado para a preservação das amostras. Na Figura 1 pode ser observado que não há perda apreciável de arsênio na presença de quantidades crescentes de cloreto, quando a amostra é preservada em meio nítrico $0,32 \mathrm{~mol} \mathrm{~L}^{-1}$.

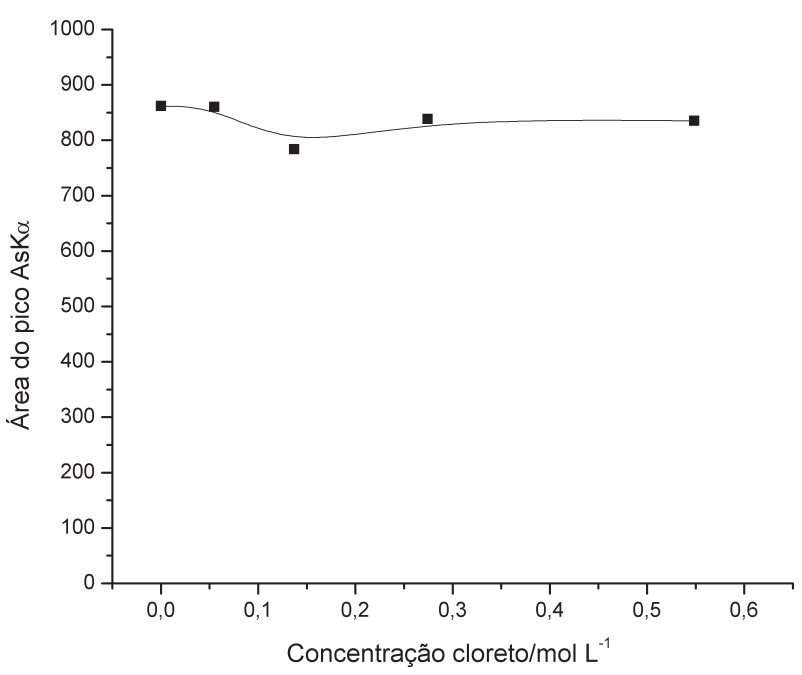

Figura 1. Influência do cloreto sobre a intensidade do sinal do As quando $0,32 \mathrm{~mol} \mathrm{~L}^{-1}$ de $\mathrm{HNO}_{3}$ é utilizado para preservação da amostra

\section{Padrão interno}

Observou-se, durante os testes iniciais, que os resultados variavam muito, o que era demonstrado pela variação do perfil do sinal analítico do arsênio. Esta variação poderia estar relacionada com a secagem não homogênea da amostra sobre a membrana, a qual levaria a diferentes concentrações do analito na região irradiada e diferenças no posicionamento da amostra frente ao feixe incidente de raios $\mathrm{X}$, que implicariam em maiores ou menores regiões irradiadas, alterando consideravelmente as concentrações finais obtidas. Decidiu-se, então, pela utilização de um padrão interno, no caso o ítrio, que geralmente é empregado para corrigir problemas que influenciam a repetitividade de um método, ou seja, a variação do sinal analítico, no caso, ocasionada pelos problemas mencionados acima. Assim, utilizando-se padrão interno, nas condições acima mencionadas, já otimizadas, a precisão dos resultados melhorou significativamente. 


\section{Parâmetros de mérito}

\section{Precisão}

Além dos fatores já mencionados que afetaram a repetitividade do método, outro parâmetro importante avaliado foi o posicionamento da amostra frente ao feixe de raios $\mathrm{X}$ incidente, que define a quantidade de amostra irradiada.

Após a etapa de secagem da amostra, esta se concentra aleatoriamente, em uma pequena área de dimensões em torno de $2 \mathrm{~mm}$ de diâmetro, sobre o filme de Mylar ${ }^{\circledR}$. O posicionamento da amostra após a secagem depende da etapa inicial de nucleação e formação dos cristais a partir da evaporação do solvente. Isso dificulta a manutenção da amostra pré-concentrada no centro da cela. Assim, amostras distribuídas aleatoriamente sobre o filme de Mylar ${ }^{\circledR}$ acabam dificultando também o correto posicionamento do feixe incidente sobre a amostra, de forma que este abranja toda a área que contém a amostra. Se o feixe atinge somente uma fração da amostra, então uma menor quantidade do analito vai ser irradiada e quantificada, o que vai diminuir a sensibilidade e a reprodutibilidade do método, muitas vezes, fornecendo resultados abaixo do limite de detecção.

Assim, após a secagem da gota da solução da amostra, faz-se necessário o acerto da posição da amostra seca sobre a membrana, no centro da cela, bem como o acerto na posição da cela no centro do suporte do espectrômetro, de forma que a radiação incidente atinja toda a amostra. Então, dois sistemas de fixação do filme de Mylar $^{\circledR}$ na cela foram utilizados: um anel fixo que não possibilitava alterar a posição da amostra na cela após a etapa de secagem e um anel flexível, que permitia o movimento da membrana Mylar ${ }^{\circledR}$, possibilitando o reposicionamento da amostra após a secagem no centro da cela. Esses dois sistemas de anéis podem ser observados na Figura 2.

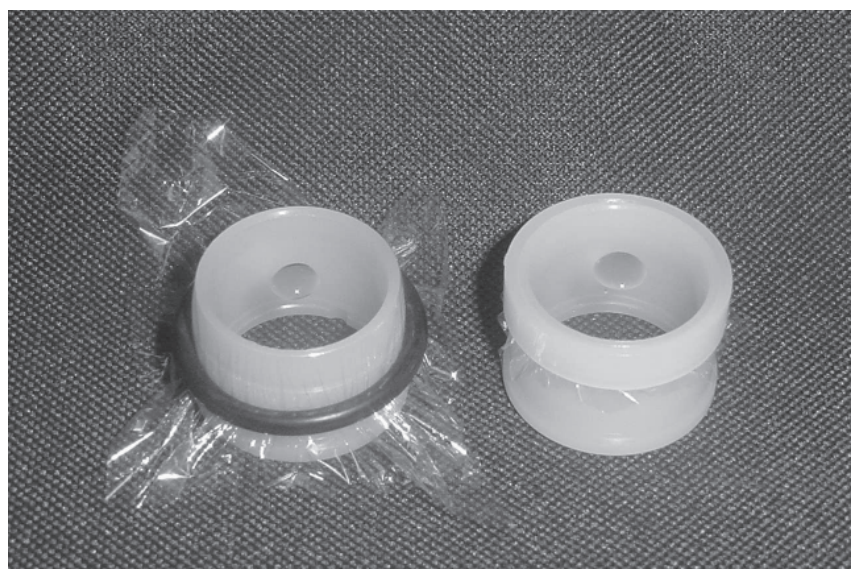

Figura 2. Amostras posicionadas na membrana, adaptadas nas celas de polietileno com anéis flexível (esquerda) e fixo (direita)

Com a utilização do Y como padrão interno e o acerto do posicionamento da amostra, tanto na cela como no equipamento, uma boa precisão foi alcançada, com desvio padrão relativo de $6,8 \%$ para $\mathrm{n}=10$, ou seja, 10 medidas de 10 replicatas de solução de As de concentração $100 \mu \mathrm{g} \mathrm{L}^{-1}$. A Figura 3 mostra um espectro típico obtido após irradiação das amostras, sendo todas as variáveis discutidas já otimizadas.

\section{Sensibilidade}

Com a utilização de $50 \mu \mathrm{L}$ de solução da amostra disposta e seca sobre a membrana de Mylar ${ }^{\circledR}$, alcançou-se um limite de detecção $(3 s)$ de $10,5 \mu \mathrm{g} \mathrm{L^{-1 }}$ de As e um limite de quantificação

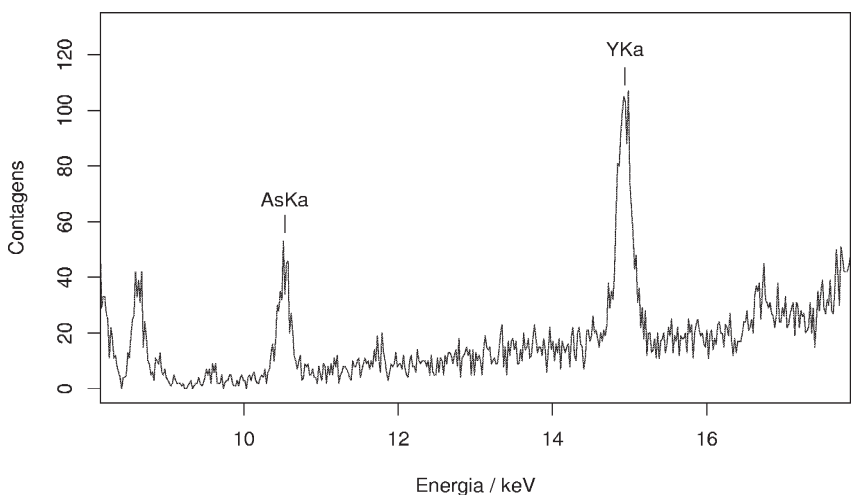

Figura 3. Espectro típico obtido para uma amostra aquosa de arsênio em forma de gota seca sobre o filme

$(10 s)$ de $65,0 \mu \mathrm{g} \mathrm{L}^{-1}$ de As, onde o $s$ é o desvio padrão de 10 replicatas do branco, composto por $\mathrm{HNO}_{3}$ e ítrio. Esses valores mostram que a técnica de fluorescência de raios $\mathrm{X}$ pode ser vista como técnica alternativa que possibilita análises quantitativas em amostras líquidas. Sbarato e colaboradores ${ }^{10}$ avaliaram a contaminação de águas subterrâneas com As, utilizando a técnica em questão. Nesse caso, os autores pré-concentraram as amostras por evaporação, chegando a $10 \%$ do volume inicial das mesmas e, posteriormente, depositaram a amostra sobre uma membrana de celofane, para secar a amostra até obterem uma matriz sólida. Em seguida, utilizaram a fluorescência de raios X com reflexão total (TXRF) para quantificar o analito. É interessante ressaltar que concentrações de As na ordem de $\mu \mathrm{g} \mathrm{L} \mathrm{L}^{-1}$ foram quantificadas, embora a etapa de pré-concentração proposta necessitou de um tempo muito longo para preparo das amostras. Outros trabalhos, como os de Bueno e colaboradores ${ }^{11}$ e Peralta-Zamora e colaboradores ${ }^{12}$, estão sendo publicados, demostrando o interesse existente em aplicar a fluorescência de raios X para quantificação de metais em amostras líquidas. O primeiro trabalho reporta a adsorção de íons cobre, geralmente presente em baixas concentrações em águas naturais, sobre carvão ativado e determinação da fase sólida por EDXRF. A grande vantagem do método proposto é a possibilidade da determinação do analito sem interferências de outros elementos como $\mathrm{Bi}^{+3} \mathrm{e}$ $\mathrm{Pb}^{+2}$, que podem estar presentes nessas amostras. No caso, os autores mostraram, no estudo de interferências, que esses elementos poderiam sim, ser quantificados simultaneamente com os íons cobre. Já o trabalho de Peralta-Zamora e colaboradores ${ }^{12}$ discute a determinação simultânea de Zr e Hf por EDXRF, após a pré-concentração dos analitos nas amostras de hidróxidos de $\mathrm{Zr}$ e de $\mathrm{Hf}$, obtidas após tratamento físico e químico de minérios de $\mathrm{Zr}$, sobre resina de troca iônica. O mérito do trabalho de Peralta-Zamora deve-se ao uso da grande vantagem da técnica, qual seja, a determinação simultânea do $\mathrm{Zr}$ e de Hf, uma vez que a determinação absoluta desses elementos por outras técnicas espectroquímicas é, geralmente, problemática, por serem elementos refratários e possuírem espectros de emissão muito complexos.

\section{Exatidão do método}

A avaliação do método desenvolvido envolveu testes de recuperação do analito e comparação dos resultados obtidos com a técnica de $\mathrm{HG} \mathrm{AAS}^{13,14,16,17}$. Dado que a sensibilidade chega em níveis de $\mu \mathrm{g} \mathrm{L}^{-1}$, o método desenvolvido pode ser comparável com outros que empregam técnicas analíticas mais sensíveis. Obteve-se uma curva analítica, utilizando padrões com concentrações de 50 a $500 \mu \mathrm{g} \mathrm{L}^{-1}$ de As, com inclinação igual a $7,14 \times 10^{-4} \mathrm{cps}_{\mu} \mathrm{A}^{-1} \mu \mathrm{g}^{-1} \mathrm{~L}$ e coeficiente de correlação de 0,9996 . 
Tabela 1. Resultados da análise das amostras de água provenientes da região do Quadrilátero Ferrífero

\begin{tabular}{lccc}
\hline Amostra & $\begin{array}{c}\text { Concentração de } \\
\text { As, } \mu \text { L L }^{-1}\end{array}$ & $\begin{array}{c}\text { Recuperação do As nas } \\
\text { amostras enriquecidas (\%) }\end{array}$ & $\begin{array}{c}\text { Concentração de As } \\
\text { medida por HG AAS } \mu g \text { L }^{-1}\end{array}$ \\
\hline Bica 1 $^{a}$ & $102 \pm 7$ & 103 & $113 \pm 11$ \\
Mina 1 $^{a}$ & $242 \pm 32$ & 105 & $260 \pm 6$ \\
${\text { Ribeirão } 1^{a}}^{a}$ ADT $^{a}$ & $225 \pm 21$ & 114 & $200 \pm 14$ \\
Mina 2 $^{b, c}$ & $920 \pm 32$ & 100 & $700 \pm 96$ \\
Barragem 1 de usina $^{b, c}$ & $1767 \pm 175$ & 102 & - \\
Barragem 2 de usina $^{b, c}$ & $2040 \pm 61$ & 104 & - \\
\hline
\end{tabular}

${ }^{a}$ Amostras enriquecidas com $100 \mu \mathrm{g} \mathrm{L}{ }^{-1}$ de As. ${ }^{b}$ Amostras enriquecidas com $1000 \mu \mathrm{g} \mathrm{L}^{-1}$ de As. ${ }^{c}$ Amostras não analisadas por HG AAS devido à alta concentração do analito. As incertezas são os desvios padrão das medidas $(\mathrm{n}=3)$.

Os resultados obtidos nas análises das amostras de águas provenientes do Quadrilátero Ferrifero estão mostrados na Tabela 1. Observa-se nessa tabela que as recuperações do As estão sempre em torno de $100 \%$, quando se analisa a recuperação do analito pelo método proposto e que os resultados obtidos são concordantes entre si para a maioria das amostras, comparando-se as duas técnicas utilizadas. Com relação à amostra ADIT, que apresentou menores concentrações de As, usando-se a HG AAS como técnica de análise, deve ser ressaltado que esta sofreu grande diluição, piorando a precisão e a exatidão das medidas. Além disso, essa amostra poderia conter altas concentrações de As (V) e, dessa forma, necessitaria de um maior tempo de pré-redução do analito para seu estado de oxidação (III), que é mais favorável à geração dos hidretos de As.

\section{CONCLUSÕES}

Os resultados obtidos com o método proposto utilizando a técnica de fluorescência de raios X evidenciam as grandes vantagens dessa técnica em análises de rotina. $\mathrm{O}$ método proporciona a determinação de arsênio em níveis de $\mu \mathrm{g} \mathrm{L}^{-1}$, sem necessidade de um preparo laborioso das amostras de água, o que leva a análises rápidas e eficientes, com baixo consumo de reagentes e pouca geração de resíduos. Além disso, a possibilidade de se trabalhar com pequenos volumes de amostras facilita consideravelmente o processo de coleta, armazenamento e transporte das amostras até o momento da análise. Outro fato a ser considerado é que o método se mostrou não susceptível a interferências, o que geralmente não acontece com muitas metodologias, as quais necessitam de agentes mascarantes ou sistemas alternativos para minimizar esses efeitos. Dessa forma, mais uma alternativa viável para determinação de arsênio total em amostras aquosas é proposta, o que pode ser bastante aplicável também para amostras sólidas, após solubilização.

Com relação às concentrações de arsênio medidas e relatadas neste trabalho, utilizando-se o método proposto, deve ser ressaltado que as águas da região do Quadrilátero Ferrífero se encontram altamente contaminadas por esse elemento, o que deve ser assunto de atenção das autoridades sanitárias.

\section{AGRADECIMENTOS}

À FAPESP e à FAPEMIG pelo apoio financeiro.

\section{REFERÊNCIAS}

1. Mandal, B. K.; Suzuki, K. T.; Talanta 2002, 58, 201.

2. Hanusch, K.;Grossmann, H; Herbert, K. A.; Rese, G. Em Ullmann's Encyclopedia of Industrial Chemistry; $5^{\text {th }}$ ed. rev., WCH: Weinheim, 1985 , vol. A3, p. 113.

3. Doak, G. O.; Gilbert Long, G.; Freedman, L. D. Em Encyclopedia of Chemical Technology; $3^{\text {rd }}$ ed., John Wiley \& Sons, 1978, vol. A3, p. 243.

4. Tsalev, D. L.; Zaprianov, Z. K.; Atomic Absorption Spectrometry in Occupational and Environmental Health Practice, CRC Press Inc.: Boca Raton, 1985, vol. 1.

5. Borba, R. P.; Tese de Doutorado, Universidade Estadual de Campinas, Brasil, 2002.

6. Borba, R. P.; Figueiredo, B. R.; Rawlins, B.; Matschullat, J.; Rev. Bras. Geociênc. 2000, 30, 554.

7. Smith, A. H.; Lingas, E. O.; Rahman, M.; Bull. World Health Organiz. 2000, 78, 1093.

8. ANVISA, Portaria no 1.469 , de 29 de dezembro de 2000, http:// www.anvisa.gov.br/legis/portarias/1469_00.htm, acessada em Janeiro 2004.

9. Standard methods for the examination of water and wastewater, 20 th ed., APHA: Washington, 1998, p. 3-32.

10. Sbarato, V. M.; Sánchez, H. J.; Appl. Radiat Isot. 2001, 54, 737.

11. Bueno, M. I. M. S.; do Amaral, L. C.; Quim. Nova 1998, 21, 434.

12. Peralta-Zamora, P.; Cornejo-Ponce, L.; Bueno, M. I. M. S.; Martins, J. W.; Talanta 1997, 44, 811.

13. Bernasconi, G. A.; Axil; Analysis of X-ray spectra by Iterative Least-squares fitting, IAEA Laboratories Seibersdorf: Austria, 1995.

14. CETESB; Guia de coleta e preservação de amostras de água, São Paulo, CETESB, 1988

15. Bortoleto, G. G.; Dissertação de Mestrado, Universidade Estadual de Campinas, Brasil, 2003.

16. Moretto, A. L.; Tese de Doutorado, Universidade Estadual de Campinas, Brasil, 2001.

17. Sthong, F.; Handbook of Chemistrty and Physics, $45^{\text {th }}$ ed., The Chemical Rubber CO: USA, 1964. 